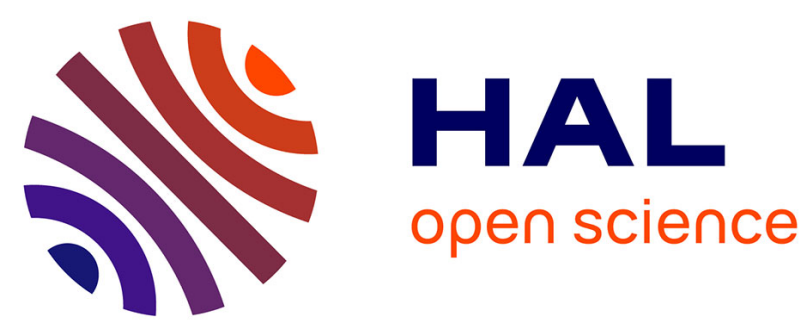

\title{
Drosophila central brain formation requires Robo proteins.
}

\author{
Emmanuelle Nicolas, Thomas Préat
}

\section{To cite this version:}

Emmanuelle Nicolas, Thomas Préat. Drosophila central brain formation requires Robo proteins.. Development Genes and Evolution, 2005, 215, pp.530-536. 10.1007/s00427-005-0009-8 . hal-00007534

\section{HAL Id: hal-00007534 https://hal.science/hal-00007534}

Submitted on 3 Oct 2005

HAL is a multi-disciplinary open access archive for the deposit and dissemination of scientific research documents, whether they are published or not. The documents may come from teaching and research institutions in France or abroad, or from public or private research centers.
L'archive ouverte pluridisciplinaire HAL, est destinée au dépôt et à la diffusion de documents scientifiques de niveau recherche, publiés ou non, émanant des établissements d'enseignement et de recherche français ou étrangers, des laboratoires publics ou privés. 


\section{Drosophila central brain formation requires Robo proteins.}

Emmanuelle Nicolas ${ }^{12 *}$ and Thomas Preat ${ }^{1}$

${ }^{1}$ Développement, Evolution, Plasticité du Système Nerveux, CNRS, 1 avenue de la Terrasse, 91190 Gif-sur-Yvette, France

${ }^{2}$ Present address :Institut Jacques Monod, 2 place Jussieu, tour 42/43, F-75251 Paris Cedex 05

*To whom correspondence should be addressed. Tel: +33 (0)1 442728 14; Fax : +33 (0)1 442752 65.nicolas@ijm.jussieu.fr 
ABSTRACT : The Robo proteins have been extensively studied in the Drosophila embryonic ventral nerve cord, in which their expression level controls the midline crossing and optic lobe formation, but nothing is known about their activities during adult central brain formation. We have analyzed how Robo guidance cues influence central complex $(\mathrm{CX})$ and mushroom body (MB) formation. Mutations of robo2 and robo3 confer a series of strong MB and CX defects. We found that the Robo2 and Robo3 proteins are expressed in two structures of the developing CX, the fan-shaped body and the noduli, and by fibers across the central neuropile. We conclude that the Robo2 and Robo3 receptors play postembryonic roles during central brain formation.

Keywords : brain, mushroom body, Drosophila, metamorphosis, Robo

\section{INTRODUCTION}

While much progress has been made in understanding the mechanisms of axon guidance in the Drosophila embryonic ventral nerve cord, little is known about the regulation of axon projection at later developmental stages. Recently, attention has turned to the development of the adult central brain (Crittenden et al., 1998; Kurusu et al., 2002; Lee et al., 1999; Boquet et al., 2000; Wang et al., 2002). Three important factors complicate this analysis: 1) the brain is composed of more than one hundred thousand neurons that form sophisticated patterns; 2) it is drastically remodeled at metamorphosis, so that axonal pathfinding takes place in a dense and disordered environment; and 3) it consists of diverse segments, unlike the ventral nerve cord, which is constructed from homogeneous units. Therefore, the development of the brain cannot be controlled by simple periodic signals but must entail sequential events of varying complexity. In the past decade an array of receptors and ligands that control growth cone pathfinding has been identified (see for review Kaprielian et al., 2001; Jacobs et al., 2000). Recently, the possibility has been raised that guidance cues generated and transmitted by these molecules have a post-embryonic role in defining neuronal structures (Godenschwege et al., 2002). The extensively studied Drosophila Robo proteins (Robo2 and Robo3) are members of a family of receptors that convey pathfinding information. Together with their ligand Slit, they control the midline crossing of embryonic ventral nerve cord axons (Rajagopalan et al., 2000a, 2000b; Simpson et al., 2000a, 2000b). Specifically, longitudinal axons expressing high levels of Robo proteins avoid Slitsecreting midline glial cells. Once axons have crossed the midline, different combinations of the three Robo receptors specify lateral positioning within the longitudinal tract.

The central complex (CX), implicated in the control of walking (Strauss and Heisenberg, 1993), lies in the middle of the brain between the MB pedunculi and connects the left and right hemispheres. The Drosophila CX, which differentiates during metamorphosis from interhemispheric structures (Hanesch, 1989; Truman et al., 1993), consists of four substructures (Fig. 1A): (1) the protocerebral bridge (PB), which lies between the MB calyces; (2) the fan-shaped body (FB), an ordered structure of horizontal layers and vertical segments; (3) the doughnut-shaped ellipsoid body (EB), which lies just anterior to the FB; and (4) the paired noduli (NO), ventral to the FB. These substructures are interconnected by sophisticated fiber projections (Hanesch, 1989)..

The mushroom bodies (MBs) are a bilateral structure in the insect brain involved in learning and memory (Davis, 1993; Heisenberg, 1998; Pascual and Preat, 2001). In adult Drosophila each MB is composed of 2500 neurons, called Kenyon cells, whose cell bodies are located in the posterior cortex and whose dendrites extend into the calyx. Their axons fasciculate into a thick bundle, the peduncle, which splits into five different lobes in the anterior part of the brain: two types of neuron branch to give rise to vertical and median lobes $\left(\alpha / \beta\right.$ and $\alpha^{\prime} / \beta^{\prime}$ lobes respectively), while a third type forms the $\gamma$ median projections (Fig. 3A) (Lee et al., 1999; Strausfeld et al., 2003; Noveen et al., 2000). Although the overall morphology of the MB is conserved through metamorphosis, larval axons degenerate and new projections develop, and newly differentiated neurons associate with modified larval neurons in the elaboration of the adult MB. The structure of the MB has functional significance, as demonstrated by our earlier work showing that $\alpha$ and/or $\alpha$ ' vertical lobes are specifically involved in long-term 
memory (Pascual and Preat, 2001; Isabel et al. 2004), but little is known about the mechanisms that guide axons during MB development (Wang et al., 2002).

In this study, we assessed the role of the Robo proteins in the formation of central brain structures. We show that (i) Robo2 and Robo3 are expressed in the subset of CX structures from larval to pupal stages; they are expressed in the FB and NO but not in the EB. Nor are they expressed in the MB. (ii) the absence of Robo proteins leads to strong $C X$ and $M B$ defects.

\section{EXPERIMENTAL PROCEDURES}

\section{Fly stocks}

Two robol alleles (Kidd et al., 1998), robo ${ }^{G A 285}$ and robo ${ }^{Z 570}$, were tested in trans. All of the following robo2 allelic combinations (Rajagopalan et al., 2000a, 2000b) conferred similar phenotypes: robo $2^{4} /$ robo $^{5}$ (11.6\% of animals were escapers); robo $2^{1} /$ robo $^{5}$ (5.2\% escapers); robo $2^{1} /$ robo $^{4}$ (1.8\% escapers). robo3 $3^{l}$ (Rajagopalan et al., 2000a; 2000b) homozygotes (14\% viability) were recovered from a balanced stock.

\section{Brain sectioning and immunohistochemistry}

Adult brain paraffin sections were generated according to (Heisenberg and Boehl, 1979). Larvae were fixed $2 \mathrm{~h}$ in Carnoy solution ( $6 \mathrm{v}$ ethanol $100 \%, 3 \mathrm{v}$ chloroform, $1 \mathrm{v}$ acetic acid), washed three times 30 min. in ethanol, incubated $\mathrm{O} / \mathrm{N}$ in methyl benzoate and embedded in paraffin blocs. Animals were cut in $10 \mathrm{vm}$ serial frontal sections that were deposited on slides and dehydrated according to standard procedures.)Sections were blocked with $10 \%$ normal goat serum (NGS) in $0.3 \%$ TBS Triton (TBT) for $20 \mathrm{~min}$ and stained with rabbit anti-Robo2 (1:50), mouse anti-Robo3 (1:5), mouse anti-FasII (1/10) (Hybridoma Bank, University of Iowa) or rabbit anti-DCO (1:1000) antibodies. Secondary antibodies conjugated to Biotin or Alexa 594 or 488 (Interchim) were used at a dilution of 1:250 for 1hr. All antibodies were diluted in $10 \%$ NGS in TBT. Stained specimens were inspected with a Leica microscope or a Leica TCS SP2 laser scanning confocal microscope (Wetzlar, Germany). Peduncle circumferences were measured with the Pegasus program (2i system, France).

\section{RESULTS and DISCUSSION}

\section{Robo mutations result in CX structural defects}

To assess whether Robo receptors have a post-embryonic role in central brain formation, we first looked for defects in the CX, which is established in the first $48 \mathrm{~h}$ after puparium formation (APF) (Hanesch et al., 1998; Truman et al., 1993). While robo3 ${ }^{l}$ amorphic flies can be recovered (Rajagopalan et al., 2000a), we found that combinations of different amorphic alleles of either robo or robo2, previously described as lethal mutations (Seeger et al., 1993 ; Battye et al., 2001), allowed us to recover adult escapers. We observed that the absence of Robo did not cause obvious anatomical defects (data not shown), whereas the absence of Robo2 and Robo3 gave rise to brain phenotypes.

We then checked the effect of loss of function mutations to determine the role of Robo proteins in central brain development. We examined frontal paraffin sections of robo2 and robo3 brains and observed major $\mathrm{CX}$ anatomical defects. The EB was abnormal, either open or completely disrupted in robo 2 and robo3 mutants (Fig.1B). In addition, the FB was split at the midline more frequently in robo 2 than in robo3 background. In robo 2 mutants, the penetrance of EB and FB defects depends on the allelic combination (Fig. 1B). None of these phenotypes was observed in wild-type flies $(n>1000)$. We observed differences between robo 2 allelic combinations: robo $2^{1} / r o b o 2^{5}$ flies exhibited the most drastic EB defects while the EB of $r o b o 2^{4} / r_{o b o} 2^{5}$ mutants was more mildly disorganised. For each 
allelic combination the penetrance of these defects correlated well with the percentage of escapers recovered: $11.6 \%$ for $r o b o 2^{1} / \mathrm{robo}^{4}, 5.2 \%$ for $r o b o 2^{1} / \mathrm{robo}^{5}$ and $1.8 \%$ for $r o b o 2^{4} / \mathrm{robo}^{5}$ flies. The FB consists of eight segments along the left-right axis, each of which contains two domains, thereby dividing the FB into 16 glomeruli (Hanesch, 1989). We took advantage of two Gal4 insertions (Boquet et al., 2000a) to analyze the effect of a Robo2 loss-of-function mutation on FB structural organisation. Gal1075 was expressed in each of the eight segments (Fig. 1C, dashed lines) and may label the socalled "pontine axons" (Hanesch et al., 1989), whose cell bodies lie in the caudal cortex and whose fibers extend horizontally towards the FB. When these axons enter the FB, one branch projects ipsilaterally while the other crosses the midline and projects into the other half of the FB. In the absence of Robo2, FB organisation collapsed: axons were concentrated at the midline region, and the segments defined by Gal1075 expression appeared to be fused (Fig. 1D). In wild type flies, axons stained by Gal1858 projected through the FB (Fig. 1E) and ramified in the EB to form a ring-like pattern. In robo 2 mutants this round shape-structure does not exist anymore despite 1858 expressingaxons seemed to still project through the FB (Fig. 1F).

\section{Robo receptors are expressed by FB axons.}

We used antibodies to determine Robo expression patterns during larval and pupal stages. Indeed, these proteins are detected in the CX as soon as axons project (Hanesch, 1989; Truman et al., 1993). At 12h APF their expression profile was similar to what is observed in third instar larvae (data not shown). By 18h APF Robo2 (Fig. 2A) and Robo3 (Fig. 2C) were detected throughout the FB. In the NO Robo2 was already present at 18h (Fig. 2B) and Robo3 at 24h (Fig 2F). From 24 to $48 \mathrm{~h} \mathrm{APF} \mathrm{(Fig.} \mathrm{2D-H),} \mathrm{the}$ two proteins are expressed. They colocalized (Fig. 2G) but were concentrated to the ventral region of the FB. By 72h Robo2 expression was restricted to the tips of the FB and the NO and Robo3 was no longer detected (data not shown). Neither Robo2 nor Robo3 was expressed in the EB (data not shown). We hypothesize that the Robo $2 / 3$ patterns of expression subdivide the FB into domains specified by a Robo code, similar to the compartmentalization of the embryonic CNS (Rajagopalan, et al., 2000a, 2000b; Simpson et al., 2000a, 2000b). Thus, this pattern defines sub-regions within the FB consisting of Robo2+Robo3 stripes within a large Robo2 area. Axons expressing the two Robo proteins project more ventrally and terminate in the NO center, while those expressing only Robo 2 project more dorsally within the FB and to the periphery of the NO. How can the loss-of-function EB defects be explained, given that Robo2 and Robo3 are not detected in the EB? FB neurons do not participate in the formation of the EB. But some of the neurons projecting in the EB pass through the FB (Fig. 1E). On their route, they may receive guidance information in the FB, provided for instance by Robo 2 and Robo3. In absence of this information, axons might not take the appropriate route to the EB.

\section{Robo mutations affect MB structural organisation}

We also investigated whether the absence of Robo proteins affects MB organization. While the absence of Robo1 did not confer major anatomical defects (data not shown), the lack of Robo2 and Robo3 gave rise to a previously undescribed MB phenotype. We sectioned adult brains and stained them with the anti-FasII antibody, which strongly labels the $\alpha / \beta$ projections, weakly labels the $\gamma$ axons but does not recognize the $\alpha^{\prime} / \beta$ ' fibers (Fig. 3A). The major defect observed in the robo 2 mutant was a partial (Fig. 3D) or complete (Fig. 3B) loss of dorsal projections (see Table 1 for penetrance). Those fibers that did not project dorsally followed a median route parallel to their $\beta$ counterparts (Fig. 3E). These results show that in absence of Robo2 dorsal lobes are not formed correctly. In addition to this projection defect, the peduncle diameter was clearly greater in robo2 mutants than in wild-type brains $(>30 \%)$ (See Fig. 3 legend). We also prepared horizontal sections to determine whether mispositioning also occurred along the antero-posterior axis. Indeed, the median lobes of robo2 mutants were positioned more posteriorly (Fig. 3F) than those of wild-type animals (Fig. 3C).

In addition to expression in the CX, Robo2 and Robo3 are detected in fibers distributed throughout the central brain (Tayler et al., 2004), forming a net through which the MB peduncle projects (Fig. 3G). 
The absence of Robo 2 expression near the peduncle may correlate with an increase in peduncle width (Fig. 3B). Robo2 was detected in the interhemispheric region on a structure that connects the two hemispheres and lies behind the MB median lobes (Fig. 3H, I). In addition, Robo2 was also expressed by a few fibers surrounding the median lobes (Fig. 3J). The positions of Robo2-expressing cells are consistent with the loss-of-function phenotype, suggesting that Robo2 might be involved in MB lobe projection. Alternatively, the mispositioning of $\mathrm{MB}$ axons might be a secondary consequence of $\mathrm{CX}$ defects. However, of brains that exhibit MB defects, $23 \%$ have defects only in the MB, indicating that $\mathrm{CX}$ structural defects do not cause MB misprojection. Robo2 expression was detected from the second instar to $48 \mathrm{~h} \mathrm{APF}$ and appeared normal in robo3 mutants (data not shown).

MB defects were also observed in robo3 mutants compared to wild type (Fig. 4A, B): (i) overextension of the tip of the dorsal lobe towards the interhemispheric region (Fig. 4C, E), (ii) the absence of $\beta$ fibers ( Fig. 4C, E) and (iii) the upwardsprojection of $\gamma$ fibers (Fig. 4D, E). We noted that the complete absence of $\beta$ projections was associated with strong $\gamma$ defects. Despite these severe defects there was no change in the size of the peduncle. In addition, there was no correlation between $\mathrm{CX}$ and MB defects in robo3 mutants: MB lobes could be strongly affected while the CX was normal (Fig. 4F) (30\% of robo3 brains exhibited MB defects but a wild-type EB), indicating that the projection defects are intrinsic to the MB.

Robo3 was expressed throughout the central brain and was observed in fibers that run close to the peduncle (Fig. 4G, H, N). The receptor was also present in a bundle of fibers that join the two hemispheres (Fig. 4I), which was distinct from a Robo2-positive bundle (Fig. 4J). This structure was positioned above the median lobes, which is consistent with the observed robo3 phenotype. Thus, we propose that Robo3 might play a role in correct positioning of $\gamma$ projections. But we do not know so far whether it is a direct role or not. Interestingly, in a 3D reconstruction we observed Robo3-positive fibers connecting the tip of dorsal MB lobe (Fig. 4L, M). We observed that $\alpha$ axons migrated too far in robo3 mutants (Fig. 4C, E, arrowhead). Thus in wild-type animals Robo3 fibers might provide a repulsive signal that prevents $\mathrm{MB}$ dorsal lobes from growing further. Like Robo2, Robo3-positive fibers were detected from the second instar to $48 \mathrm{~h}$ APF and appeared normal in robo2 mutants (data not shown). Significantly, larval MB projections were normal in robo2 and robo3 mutants (data not shown), indicating that these genes play a role when adult MB projections form at metamorphosis but not during MB embryonic development.

The robo 2 mutant exhibits less severe brain defects than does the robo3 mutant, while the robo mutant has apparently normal MBs. The converse situation is observed in embryos (Rajagopalan et al., 2000a; Simpson et al., 2000b), in that robo mutants exhibit the strongest mutant phenotypes while robo3 mutants display very mild defects. Thus, Robo proteins appear to have acquired specialized developmental functions: Robo is the main partner for Slit in the embryo, while Robo2 and Robo3 are the major players at metamorphosis. Their patterns of expression and their loss of function phenotypes are indicative of a role in $\mathrm{CX} / \mathrm{MB}$ morphogenesis and development. The molecular and cellular roles of Robo proteins during metamorphosis remain to be defined. In particular, it is not known if Robo proteins are directly required for $\mathrm{MB}$ and $\mathrm{CX}$ formation, or if their primary role is to control the differentiation of Robo-expressing structures.

\section{ACKNOWLEDGMENTS}

We thank B. J. Dickson for providing Robo2 and Robo3 antibodies and robo2 and robo3 fly stocks; R. L. Davis for the anti-DCO antibody; S. Brown and C. Talbot for confocal microscopy expertise; G. Isabel, G. Didelot and A. Pascual for their fruitful comments on the manuscript. This work was supported by research grants from the Association pour la Recherche contre le Cancer, the Ligue contre le Cancer and the Human Frontier Science Program. 


\section{REFERENCES:}

Bagri A, Marin O, Plump AS, Mak J, Pleasure SJ, Rubenstein JL, Tessier-Lavigne M (2002) Slit proteins prevent midline crossing and determine the dorsoventral position of major axonal pathways in the mammalian forebrain. Neuron 33:233-248.

Battye R, Stevens A, Perry RL, Jacobs JR (2001) Repellent signaling by Slit requires the leucine-rich repeats. J Neurosci 21:4290-4298.

Boquet I, Hitier R, Dumas M, Chaminade M, Preat T (2000) Central brain postembryonic development in Drosophila: implication of genes expressed at the interhemispheric junction. J Neurobiol 42:33-48.

Crittenden JR, Skoulakis EM, Han KA, Kalderon D, Davis RL (1998) Tripartite mushroom body architecture revealed by antigenic markers. Learn Mem 5:38-51.

Davis RL (1993) Mushroom bodies and Drosophila learning. Neuron 11:1-14. Godenschwege TA, Simpson JH, Shan X, Bashaw GJ, Goodman CS, Murphey RK. (2002) Ectopic expression in the giant fiber system of Drosophila reveals distinct roles for roundabout (Robo), Robo2, and Robo3 in dendritic guidance and synaptic connectivity. J Neurosci. 22(8):3117-29.

Hanesch U., Fischbarch KF, Heisenberg M. (1989). Neural architecture of the central complex in Drosophila melanogaster. Cell Tissue Res 457:343-366.

Heisenberg M and Boehl K (1979) Isolation of anatomical brain mutants of Drosophila by histological means. Z. Naturforsh. B:143-147.

Heisenberg M (1998) What do the mushroom bodies do for the insect brain? an introduction. Learn Mem 5:1-10.

Hidalgo A and Brand AH (1997) Targeted neuronal ablation: the role of pioneer neurons in guidance and fasciculation in the CNS of Drosophila. Development 124:3253-3262.

Isabel, I. Pascual, A. and Preat, T. (2004). Exclusive consolidated memory phases in Drosophila. Science 304 : 1024-1027.

Jacobs JR (2000) The midline glia of Drosophila: a molecular genetic model for the developmental functions of glia. Prog Neurobiol 62:475-508.

Kaprielian Z, Runko E, Imondi R (2001) Axon guidance at the midline choice point. Dev Dyn 221:154-181.

Keynes R, Tannahill D, Morgenstern DA, Johnson AR, Cook GM, Pini A (1997) Surround repulsion of spinal sensory axons in higher vertebrate embryos. Neuron 18:889-897.

Kidd T, Brose K, Mitchell KJ, Fetter RD, Tessier-Lavigne M, Goodman CS, Tear G (1998) Roundabout controls axon crossing of the CNS midline and defines a novel subfamily of evolutionarily conserved guidance receptors. Cell 92:205-215.

Kurusu M, Awasaki T, Masuda-Nakagawa LM, Kawauchi H, Ito K, Furukubo-Tokunaga K (2002) Embryonic and larval development of the Drosophila mushroom bodies: concentric layer subdivisions and the role of fasciclin II. Development 129:409-419.

Lee T, Lee A, Luo L (1999) Development of the Drosophila mushroom bodies: sequential generation of three distinct types of neurons from a neuroblast. Development 126:40654076.

Noveen A, Daniel A, Hartenstein V (2000) Early development of the Drosophila mushroom body: the roles of eyeless and dachshund. Development 127:3475-3488.

Pascual A and Preat T (2001) Localization of long-term memory within the Drosophila mushroom body. Science 294:1115-1117.

Rajagopalan S, Nicolas E, Vivancos V, Berger J, Dickson BJ (2000a) Crossing the Midline. Roles and Regulation of Robo Receptors. Neuron 28:767-777.

Rajagopalan S, Vivancos V, Nicolas E, Dickson BJ (2000b) Selecting a Longitudinal Pathway. Robo Receptors Specify the Lateral Position of Axons in the Drosophila CNS. Cell 103:1033-1045.

Seeger M, Tear G, Ferres-Marco D, Goodman, CS (1993) Mutations affecting growth cone guidance in Drosophila: genes necessary for guidance toward or away from the midline. Neuron 10:409-426

Shu T, Richards LJ (2001) Cortical axon guidance by the glial wedge during the development of the corpus callosum. J Neurosci. 21:2749-2758. 
Simpson JH, Bland KS, Fetter RD, Goodman CS (2000a) Short-Range and Long-Range Guidance by Slit and Its Robo Receptors. A Combinatorial Code of Robo Receptors Controls Lateral Position. Cell 103:1019-1032.

Simpson JH, Kidd T, Bland KS, Goodman CS (2000b) Short-range and long-range guidance by Slit and its Robo receptors. Robo and Robo2 play distinct roles in midline guidance. Neuron 28:753-766.

Strauss R and Heisenberg M (1993) A higher control center of locomotor behavior in the Drosophila brain. J Neurosci. May;13(5):1852-61.

Strausfeld NJ, Sinakevitch I, Vilinsky I (2003) The mushroom bodies of Drosophila melanogaster: an immunocytological and golgi study of Kenyon cell organization in the calyces and lobes. Microsc Res Tech. Oct 1;62(2):151-69.

Tayler TD, Robichaux MB, Garrity PA. (2004) Compartmentalization of visual centers in the Drosophila brain requires Slit and Robo proteins. Development. Dec;131(23):5935-45.

Truman JW, Taylor BJ, Awad TA (1993) Formation of the adult nervous system. In: The development of Drosophila melanogaster. Cold Spring Harbor, NY: Cold Spring Harbor Laboratory Press. 12451276.

Wang J, Zugates CT, Liang IH, Lee CH, Lee T (2002) Drosophila Dscam is required for divergent segregation of sister branches and suppresses ectopic bifurcation of axons. Neuron 33:559-571. 


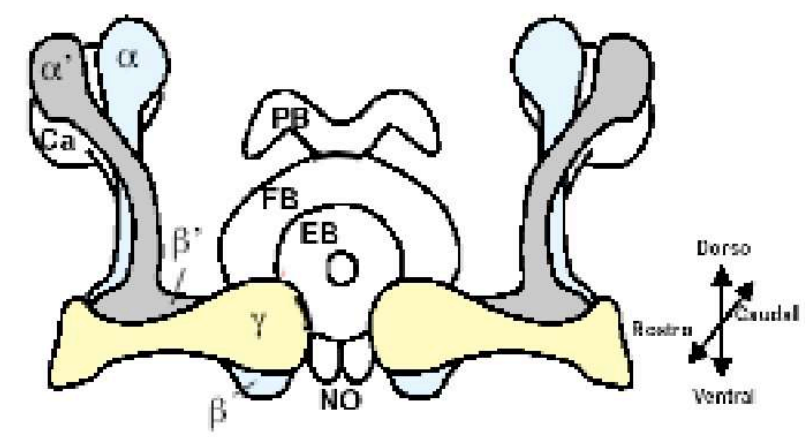

B

Elipsoid Body (EB)

Fan-shaped body (FB)

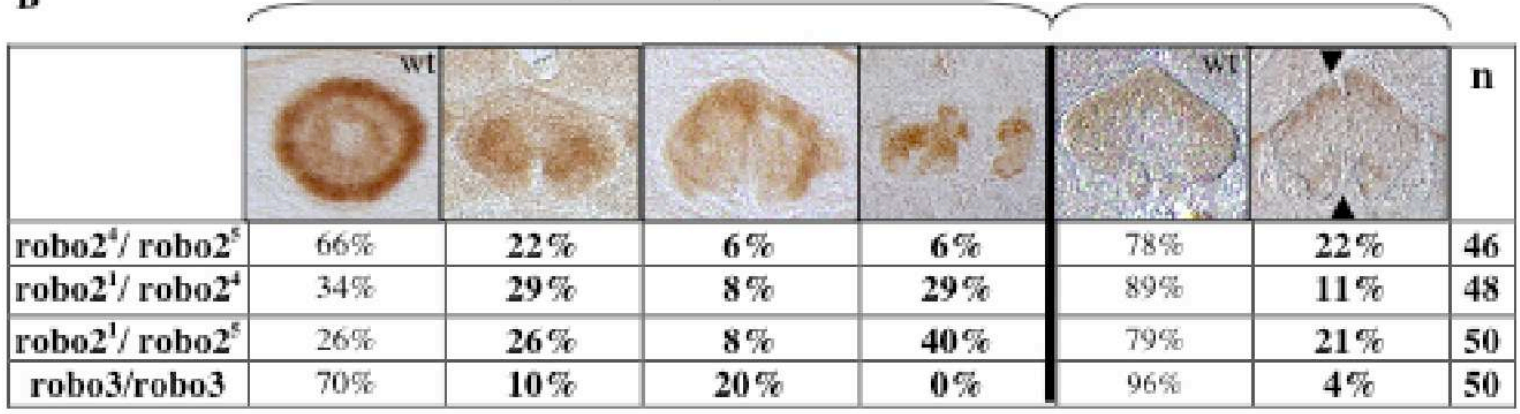

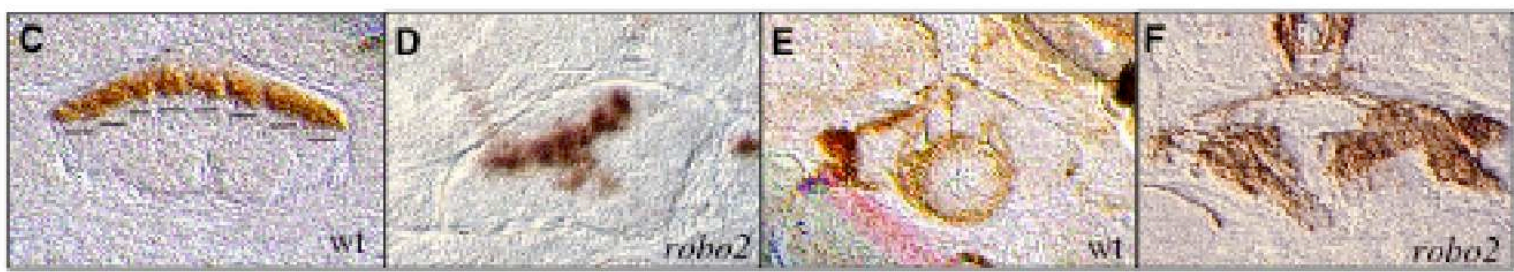

Fig. 1 The CX is disrupted in robo2 and robo 3 mutants. A In the adult, MB axons fasciculate into a thick bundle, the peduncle, which then splits into different lobes in the anterior part of the brain. Two types of neuron branch to give rise to the vertical and median lobes $\left(\alpha / \beta\right.$ and $\alpha^{\prime} / \beta$ ' lobes respectively), while a third forms the $\gamma$ median projections. The protocerebral bridge $(\mathrm{PB})$ lies between the MB calyces $(\mathrm{Ca})$. Underneath is located the fan-shaped body $(\mathrm{FB})$, an ordered structure of horizontal and vertical segments. The doughnut-shaped ellipsoid body (EB) lies anterior to the FB and the nodules (NO) are ventral to the FB. B Adult brain frontal paraffin sections at the EB and FB levels, labeled with the antiFasII antibody. The penetrance of EB and FB defects is indicated for each allelic combination. Mutant phenotypes range from a simple split in the EB to complete disorganization of the structure. None of these defects was observed in wild-type flies $(\mathrm{n}=1000)$. FB defects are mild, with bifurcation being the strongest phenotype. C Ga11075, UAS-CD8-GFP expression in the eight segments (dashed lines). D This organisation collapses at the midline in absence of Robo2. $\mathbf{E}$ Gal1858 is expressed by axons that run accross the FB and that stop in the EB. F In loss-of-function robo2 mutants these axons are misguided so that the EB cannot be formed properly. 


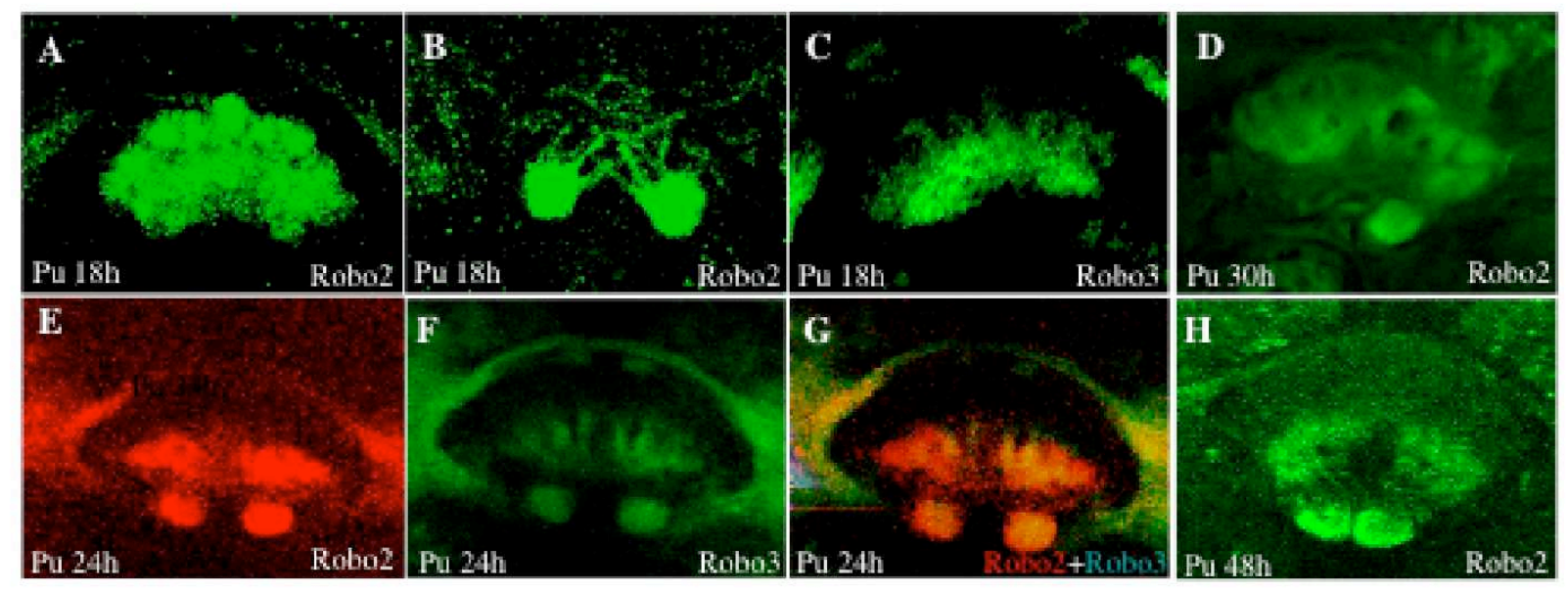

Fig. 2 Robo2 and Robo3 are expressed by the FB and by NO axons. A At $18 \mathrm{~h}$ and (E) $24 \mathrm{~h}$ APF, Robo2 is present throughout the FB and (B) is expressed by axons that project ipsilaterally and contralaterally into the NO. C Robo3 is also detected in the FB and NO, but in more restricted domains that overlap with Robo2 expression domains (F). D-H From 24h to $48 \mathrm{~h}$ APF, the Robo2 expression domain is progressively restricted to the ventral part of the FB but is still expressed by the NO. 


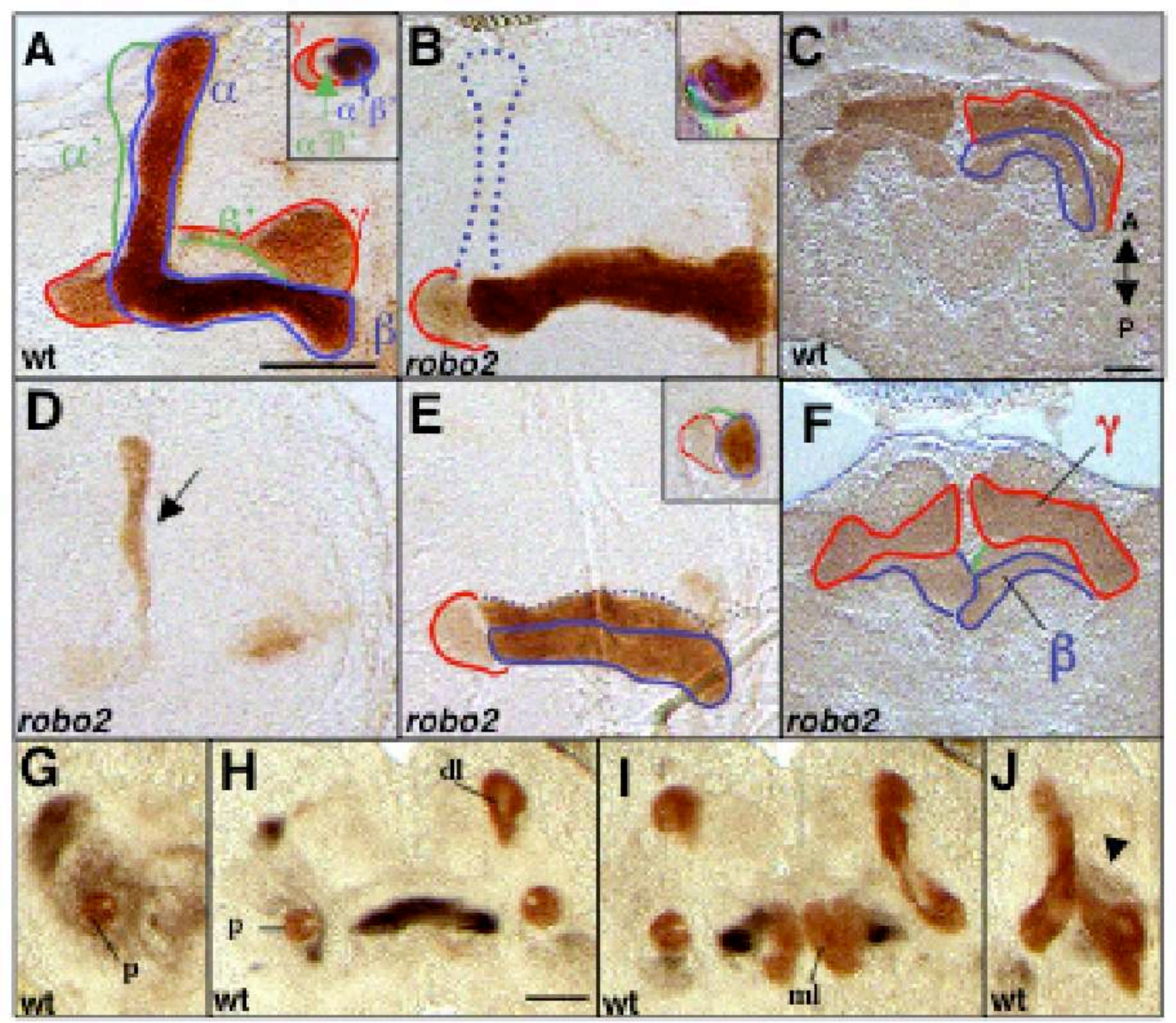

Fig. 3 The robo 2 mutation affects MB projections. A, C, G-J Wild-type, B, D-F robo $2^{1} / r o b o 2^{4}$. A Three sets of MB neurons generate five axonal lobes: the $\gamma$ lobe is outlined in red, the $\alpha / \beta$ lobes in blue and the $\alpha^{\prime} / \beta^{\prime}$ lobes in green. $\mathbf{D}$ and $\mathbf{E}$ are two consecutive sections of the same brain. We observed that in the absence of Robo2 the dorsal lobe is partly (D) or entirely absent (B). D $\alpha$ axons that do not project dorsally are recovered along the median lobe (dotted line, E). A, B, E Upper right hand boxes show peduncle frontal sections. In robo 2 mutants the peduncle is $30 \%$ larger than in wild-type animals: robo $2^{4} /$ robo $^{5}: 69.3 \pm 1.8$ arbitrary units (a.u., $\left.\mathrm{n}=99\right)$ compared to CS: $54.0 \pm 1.1$ a.u. $(\mathrm{n}=70)$; robo $2^{1} /$ robo $2^{5}: 66.0$ \pm 2.1 a.u. $(n=48)$ compared to $C S: 46.6 \pm 1.9$ a.u. $(n=24)$; robo $2^{1} /$ robo $^{4}: 67.6 \pm 3.0$ a.u. $(n=40)$ compared to $C S: 51.8 \pm 2.5$ a.u. $(\mathrm{n}=26)$. C, F Horizontal brain sections. F In robo2 mutants, axons project towards the posterior part of the brain. Scale bars: $40 \mathrm{~mm}$.

The Robo2 pattern of expression was visualized by immunohistochemical analysis of third instar larval brain paraffin sections with anti-Robo2 (brown) and anti-DCO (red) antibodies, which label the MB axons. G-J Posterior-to-anterior sections of the same brain. G Robo2-expressing (brown) fibers are distributed throughout the central brain. $\mathbf{H}$ Some of these fibers fasciculate together, forming a structure that connects the two hemispheres. I Section showing that this structure is positioned behind the median lobes. J A ring-like structure expressing Robo2 surrounds the median lobes (arrowheads). Inspection of parasaggital sections indicates that these fibers are derived from the Robo2 interhemispheric structure (data not shown). Scale bar: 40mm; p, peduncle; dl, dorsal lobe; ml, median lobe. 

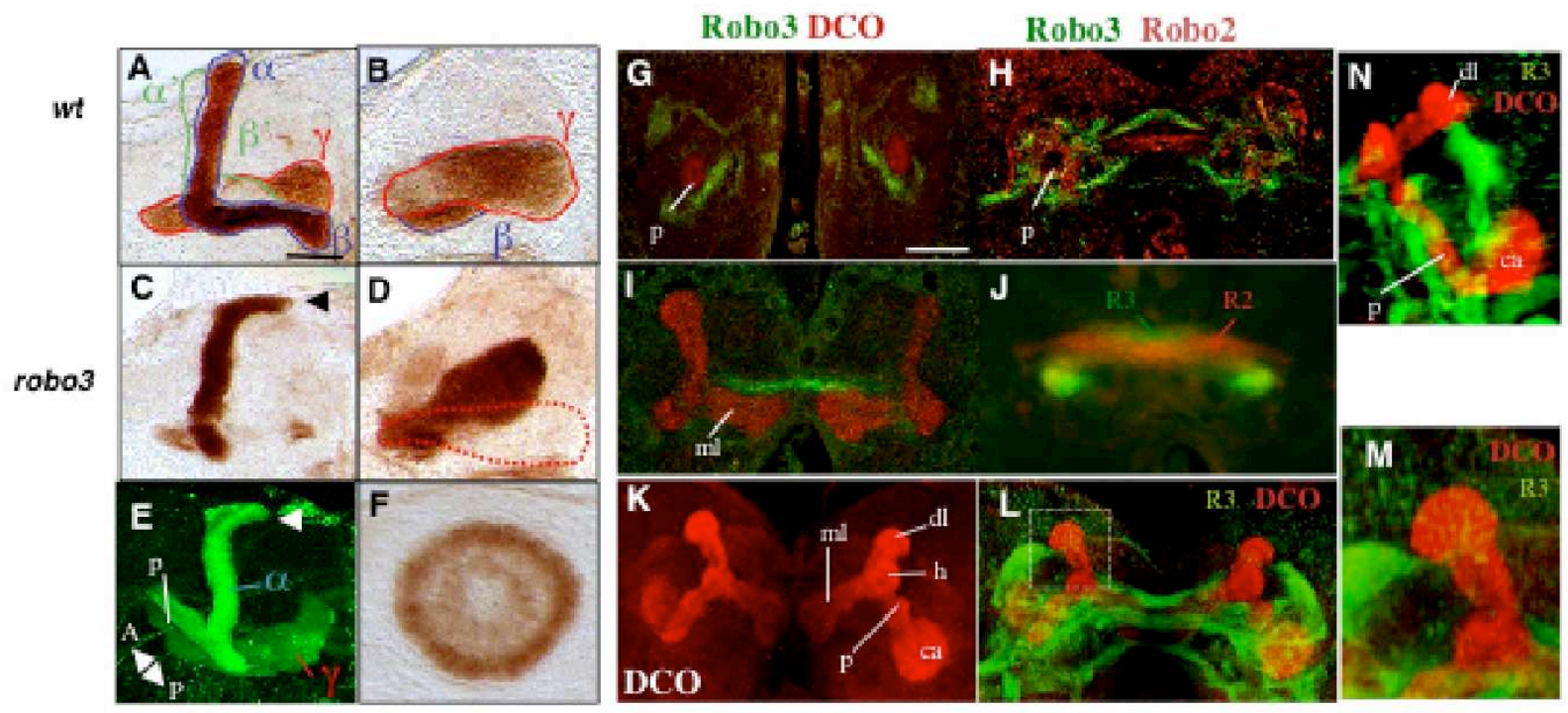

Fig. 4 Robo3 is expressed in the larval brain, and the mutation affects MB morphogenesis. A, B wild-type, C, D, F robo3 ${ }^{1} /$ robo $^{l}$, composite images of adult brain frontal paraffin sections labeled with the anti-FasII antibody. G-J composite images of wt adult brain frontal paraffin sections labeled with the anti-Robo3 + anti-DCO antibodies (G, I) and anti-Robo3 and anti-Robo2 antibodies (H, J) E, K-N 3D reconstruction of a confocal stack (Metamorph) of MB axonal projections in a robo3 mutant. A, B Three sets of MB neurons generate five axonal lobes: the $\mathrm{g}$ lobe is outlined in red, the a/b lobes in blue and the $\mathrm{a}^{\prime} / \mathrm{b}$ ' lobes in green. B Wild-type $\mathrm{g}$ lobe. $\mathbf{C}, \mathbf{E}$ In robo3 mutants a lobe is positioned closer to the midline (arrowhead), E b projections are absent and $\mathbf{D}$ the $\mathrm{g}$ lobes project dorsally outside the normal axis. The dotted line indicates the initial wild-type position $(\mathbf{B})$. F Note that the EB can be normal although the MB exhibits strong projection defects $(\mathbf{C})$ ( $\mathbf{C}$ and $\mathbf{F}$ are two sections from the same stack). Scale bar: $40 \mathrm{~mm}$. p, peduncle.

The Robo3 pattern of expression was visualized by immunohistochemical analysis of third instar larval brain paraffin sections. G, I Staining with anti-Robo3 (red) and anti-DCO (green) antibodies shows that Robo3 is expressed throughout the neuropile. H, J Staining with the anti-Robo3 (green) and anti-Robo2 (red) antibodies. H The two proteins are expressed by different fibers across the central brain. I A subset of Robo3-expressing (red) fibers accumulate dorsal to the MB median lobes, while $\mathbf{J}$ a Robo2-expressing (green) structure is located posterior to the same lobes. $\mathbf{K}, \mathbf{L} 3 \mathrm{D}$ reconstructionof MB axons stained with DCO- (red) and Robo3-specific antibodies (green, L). M A high magnification view of the scale window in $\mathbf{L}$, showing Robo3-positive fibers connecting the tips of dorsal MB projections. $\mathbf{N}$ A lateral view of a 3D projection of the right hemisphere presented in $\mathbf{L}$. Some of the Robo3-positive fibers run near the calyx, along the peduncle and at the junction of the two hemispheres (L). Scale bar: $80 \mathrm{~mm}$. Kc, Kenyon cells; ca, calyx; p, peduncle; dl, dorsal lobe; ml, median lobe. 
Table 1 : summary of anitomical MB phenotypes induced by robo2 and robo 3 mutations.

\begin{tabular}{|c|c|c|c|c|c|c|}
\hline Genotype & $\begin{array}{c}\text { Dorsal } \\
\text { lobes close } \\
\text { to the } \\
\text { midline }\end{array}$ & $\begin{array}{l}\text { Crosswise } \\
\text { projection } \\
\text { of } \gamma \text { fiber }\end{array}$ & $\begin{array}{l}\text { Absence } \\
\text { of median } \\
\text { projection }\end{array}$ & $\begin{array}{l}\text { Absence } \\
\text { of dorsal } \\
\text { projection }\end{array}$ & $\begin{array}{c}\text { Partial } \\
\text { median } \\
\text { projection }\end{array}$ & $\mathrm{N}^{*}$ \\
\hline robo $2^{2} /$ robo $2^{5}$ & - & - & . & $16 \%$ & $32 \%$ & 37 \\
\hline robo $2^{s} /$ robo $^{4}$ & - & $=$ & - & $12 \%$ & $8 \%$ & 60 \\
\hline robo $2^{\prime} / r o b o 2^{\prime}$ & - & - & - & $34 \%$ & $18 \%$ & 38 \\
\hline robos'/robos' & $100 \%$ & $73 \%$ & $48 \%$ & $=$ & - & 53 \\
\hline
\end{tabular}

* The Canton-S control never exhibited these phenotypes $(n>1000)$. 